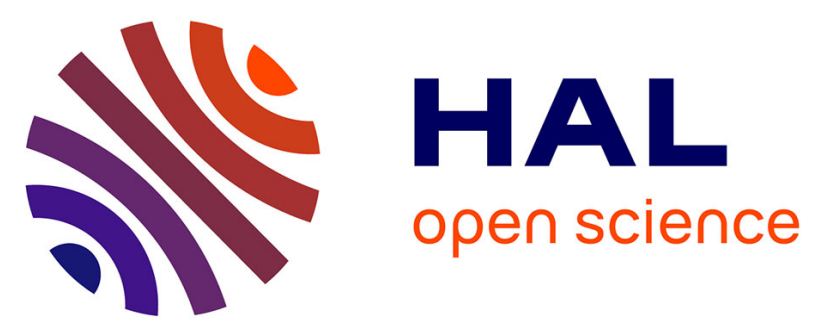

\title{
Theoretical studies of the ground state and of the spectroscopic properties of ethyl 5-amino-2-methyl-1,2- dihydro-3-phenylpyrido[3,4-b]pyrazin-7-yl carbamate analogs
}

\author{
M Sarrazin, Pascale Barbier, C Briand, V Peyrot
}

\section{To cite this version:}

M Sarrazin, Pascale Barbier, C Briand, V Peyrot. Theoretical studies of the ground state and of the spectroscopic properties of ethyl 5-amino-2-methyl-1,2-dihydro-3-phenylpyrido[3,4-b]pyrazin7-yl carbamate analogs. Journal of Molecular Structure: THEOCHEM, 2001, 541 (1-3), pp.253-262. 10.1016/S0166-1280(00)00809-5 . hal-01480677

\section{HAL Id: hal-01480677 \\ https://hal-amu.archives-ouvertes.fr/hal-01480677}

Submitted on 13 Mar 2017

HAL is a multi-disciplinary open access archive for the deposit and dissemination of scientific research documents, whether they are published or not. The documents may come from teaching and research institutions in France or abroad, or from public or private research centers.
L'archive ouverte pluridisciplinaire HAL, est destinée au dépôt et à la diffusion de documents scientifiques de niveau recherche, publiés ou non, émanant des établissements d'enseignement et de recherche français ou étrangers, des laboratoires publics ou privés. 


\title{
Theoretical studies of the ground state and of the spectroscopic properties of ethyl 5-amino-2-methyl-1,2-dihydro-3- phenylpyrido[3,4-b]pyrazin-7-yl carbamate analogs
}

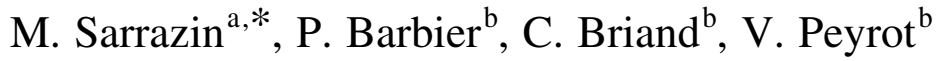 \\ ${ }^{a}$ Laboratoire de biophysique I, 27 Bd Jean Moulin, 13385 Marseille Cedex 5, France \\ ${ }^{\mathrm{b}}$ UMR CNRS 6032, 27 Bd Jean Moulin, 13385 Marseille Cedex 5, France
}

Received 19 September 2000; revised 20 October 2000; accepted 25 October 2000

\begin{abstract}
The interaction of antimitotic compounds with tubulin has been studied in detail in our laboratory; among them, the two chiral isomers of ethyl 5-amino-2-methyl-1,2-dihydro-3-phenylpyrido[3,4-b]pyrazin-7-yl carbamate, NSC $613863(R)-(+)$ and NSC 613862 (S)-(-) (CI980) and the three achiral analogs NSC 330770 (ethyl 5-amino-1,2-dihydro-3-phenylpyrido[3,4b]pyrazin-7-yl carbamate), NSC 337238 (ethyl 5-amino-3-phenylpyrido[3,4-b]pyrazin-7-yl carbamate) and C179 (ethyl 5amino-2-methyl-3-phenylpyrido[3,4-b]pyrazin-7-yl carbamate). In this study, by AM1 calculations, we have investigated the ground state $\left(\mathrm{S}_{0}\right)$, the near-UV absorption, the fluorescence emission properties of these compounds in the order to better understand the behavior of each drug and to enlighten their binding mechanism to tubulin. A modification of the ring B such as a methyl substituent or a second insaturation center drastically modified the affinity for tubulin. AM1 results indicated that ring A and B were mainly involved in the first step of binding to tubulin, the second step consisted in the interaction of the phenyl ring C. The spectra of the compounds have shown that an excited state rearrangement took place and that the molecules in the $\mathrm{S}_{1}$ state are rendered more planar. The rotation of the phenyl appeared to be an unfavorable pathway but an imino form, stabilized by an intramolecular hydrogen bond between the COO moiety of the side chain and an hydrogen at atom N6 could play a role either in the $S_{0}$ and/or in the $S_{1}$ state. (C) 2001 Elsevier Science B.V. All rights reserved.
\end{abstract}

Keywords: Tubulin; Microtubules; CI980; Semi-empirical calculations; Resonance

\section{Introduction}

The binding of the cytostatic drug colchicine to tubulin was a fundamental discovery leading to the assessment that the microtubule network is an essential component of the cytoskeleton of eucaryotic cells. Colchicine (Fig. 1) biological effects are connected

* Corresponding author. Tel.: +33-4-91-78-20-24; fax: +33-491-83-55-96.

E-mail address: marcel.sarrazin@pharmacie.univ-mrs.fr (M. Sarrazin). with specific and with high affinity to tubulin (for reviews see Ref. [1]). The development of anti-mitotic drugs has been an important therapeutic approach to the control of proliferative cell growth, and an essential component of pharmacological and biochemical studies of microtubules.

In this way, the interaction of derivative series of antimitotic compounds with a 1,2 dihydro-3-phenylpyrido[3,4-b] pyrazin-7-yl carbamate structure with tubulin has been extensively studied in our laboratory. The two chiral isomers of ethyl 5-amino-2-methyl-1,2-dihydro3-phenylpyrido[3,4-b]pyrazin-7-yl carbamate, NSC 
<smiles>COc1cc2c(c(OC)c1OC)-c1ccc(OC)c(=O)cc1[C@@H](NC(C)=O)CC2</smiles>

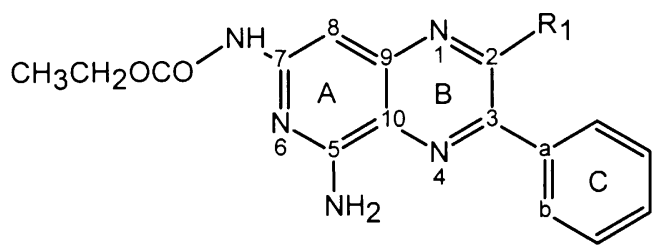

NSC $337238(U)$

C179 (T)

$$
\begin{aligned}
& \mathrm{R}_{1}=\mathrm{H} \\
& \mathrm{R}_{1}=\mathrm{CH}_{3}
\end{aligned}
$$<smiles>[R7]C1([R1])Nc2cc(NOC(=O)OC)nc(N)c2N=C1c1ccccc1</smiles>

NSC $613862(S)$

NSC $330770(V)$

$$
\begin{array}{r}
\mathrm{R}_{1}=\mathrm{CH}_{3} \quad \mathrm{R}_{2}=\mathrm{H} \\
\mathrm{R}_{1}=\mathrm{R}_{2}=\mathrm{H}
\end{array}
$$

Fig. 1. Structures of colchicine and studied compounds.

$613863(R)-(+)$ and NSC $613862(S)-(-)($ CI980)

(Fig. 1), inhibit microtubule polymerization in vitro by binding to tubulin with high affinity, overlapping ring $\mathrm{A}$ and ring $\mathrm{C}$ loci of the colchicine binding site [2]. The $S$-isomer is the most efficient [3].

As for colchicine (for reviews see Ref. [4]), the $R$ isomer displays a two-step binding mechanism: an initial binding followed by a conformational change of the initial complex [5]. The orientation of the methyl group influences the binding kinetics, since similar experiments with the $S$-isomer demonstrate a slower association and a larger activation energy for dissociation than for the $R$-isomer [5].

The characteristics of the interaction with tubulin of the three achiral analogs NSC 330770 (compound V) (ethyl 5-amino-1,2-dihydro-3-phenylpyrido[3,4-b]pyrazin-7-yl carbamate), NSC 337238 (compound U) (ethyl 5-amino-3-phenylpyrido[3,4-b]pyrazin-7-yl carbamate) and C179 (compound T) (ethyl 5-amino-2-methyl-3phenylpyrido[3,4- $b$ ]pyrazin-7-yl carbamate) (Fig. 1) indicate that a large dipole moment, a 1,2-dihydro structure and an electronic density localized on the C3, N4, and C10 atoms lead to a strong activity [6]. Moreover, in aqueous solution the $R$ - and $S$-isomers are characterized by identical Raman spectra, whereas upon binding with tubulin the Raman spectra of the $R$ and $S$-isomers are different [7].

In a recent work focused on the effects of recognition by tubulin on the ligands themselves, we have demonstrated that ligand fluorescence emission is strongly enhanced and blue shifted upon binding to tubulin [8]. Among solvent series, water had a specific solvent effect, except on the 1,2-dehydro analogs NSC 337238 (U) and C179 (T), suggesting hydrogen bonding with $\mathrm{N} 1$. The emission of tubulin-bound $R$-isomer, $S$-isomer and $\mathrm{V}$ could be mimicked by solvent viscosity, supporting the idea that the intramolecular rotation between the pyridopyrazine and phenyl rings is frozen upon binding.

In this study, by AM1 theoretical chemistry calculations, we investigated the ground state $\left(\mathrm{S}_{0}\right)$, the nearUV absorption, the fluorescence emission properties of the S, T, U and V compounds to explain the differences observed between the free and bound tubulin ligands. Furthermore, by calculations we also examined the influence of the methyl at $\mathrm{C} 2$, and the introduction of the double bond N1=C2. Finally, AM1 calculations indicated that for all compounds two 
Table 1

Total energy, heat of formation, $\Delta H_{\mathrm{f}}^{0}$ dihedral angles between ring B and phenyl group C, $\phi$, dipole moment, $\mu$, HOMO energy, $E_{\mathrm{HOMO}}, \mathrm{LUMO}$ energy, $E_{\mathrm{LuMO}}$, according to semi-empirical calculations using the AM1 method and experimental $\Delta H_{0}$ of the binding of the studied compounds to tubulin [6]

\begin{tabular}{|c|c|c|c|c|c|c|c|}
\hline & Total energy $(\mathrm{kcal} / \mathrm{mol})$ & Heat formation $(\mathrm{kcal} / \mathrm{mol})$ & $\phi\left({ }^{\circ}\right)$ & $\mu$ (Debye) & $\Delta H_{0}(\mathrm{kcal} / \mathrm{mol})$ & $E_{\text {Номо }}(\mathrm{eV})$ & $E_{\text {LUMO }}(\mathrm{eV})$ \\
\hline V & -91257.66 & 10.1026 & 21.30 & 4.28 & -13.6 & -8.1275 & -0.5712 \\
\hline S & -94849.23 & 5.3768 & 46.03 & 4.25 & -20.8 & -8.1915 & -0.4686 \\
\hline $\mathrm{U}$ & -90613.01 & 24.9448 & 42.10 & 4.01 & -7.9 & -8.4925 & -0.9249 \\
\hline $\mathrm{T}$ & -94203.60 & 21.1866 & 46.20 & 4.31 & -6.9 & -8.4839 & -0.8540 \\
\hline
\end{tabular}

imino forms I and II could exist in parallel with the amino forms. Interestingly, only for $\mathrm{S}$ and $\mathrm{V}$ compounds the calculated absorption spectra of the imino form II are closely related to the measured spectra.

\section{Material and methods}

\subsection{Semi-empirical quantum mechanical calculations}

AM1 calculations were performed with the HYPERCHEM package [9] to estimate atomic partial charges, optimize geometry of molecules, and predict energy. This program enabled structure drawing and optimization of the geometry of each species using $\mathrm{MM}+$ force field [10]. Then, the optimization of the geometry of each species was carried out using AM1 method. Each SCF calculation was carried out until the change in energy on successive iterations was less than $0.0001 \mathrm{kcal} / \mathrm{mol}$. A geometry optimization was stopped when the gradient norm in $\mathrm{kcal} / \mathrm{mol} / \AA$ was less than 0.3. Wiberg bond order, and configuration interaction calculations were performed using ARGUSLAB [11-14] to study the excited-state properties of the compounds. ArgusLab is a free molecular modeling program ${ }^{1}$ for Windows 95/98 and NT 4.0. The calculations of transition energies and oscillator strengths were carried out using the INDO1/S method [15-17]. The conformations used in the excited-state calculations were taken from ground-state structures at local energy minima found with AM1.

\footnotetext{
${ }^{1}$ Available at (http://www.seanet.com/ mthompson/ArgusLab/ index.htm).
}

\subsection{Spectroscopic measurements}

All the details of the measurements and results of the absorption and fluorescence spectra were recently published [8]. In the order to precise the $\lambda_{\max }$ and full width at half the maximum height (FWHM, $\mathrm{cm}^{-1}$ ) of the absorption spectra of the compounds diluted in sodium phosphate buffer $\mathrm{pH} 7$, a deconvolution was carried out using the SIGMAPLOT software [18].

\section{Results and discussions}

\subsection{The ground state}

The ground-state parameters: the total energy, heat of formation $\left(\Delta H_{0}\right)$, dipole moment, the dihedral angles between ring B and phenyl group C, HOMO and LUMO energies for $\mathrm{S}, \mathrm{T} \mathrm{U}$ and $\mathrm{V}$ compounds are reported in Table 1. No calculations were done concerning the $\mathrm{R}$ compound with respect to $\mathrm{S}$ compound since the semi-empirical methods are not really able to distinguish the chiral isomers. In effect, in the calculations, the molecules were considered as isolated, and all the spatial interactions responsible for the difference of reactivity are ignored.

\subsection{Dihedral angles}

Firstly, AM1 calculations showed that the dihedral angles between ring $\mathrm{B}$ and phenyl group $\mathrm{C}$ ranged from $21^{\circ}$ for $\mathrm{V}$ to about $45^{\circ}$ for $\mathrm{S}, \mathrm{T}$ and $\mathrm{U}$. For $\mathrm{S}$ and $\mathrm{T}$, the steric hindrance due to the methyl at $\mathrm{C} 2$ is responsible of these largest values. These values are higher (by a factor 2) than the previously calculated values using the CNDO/2 method [5].

For colchicine and its analogs the importance of ring $\mathrm{C}$ for binding to tubulin has been pointed out 
by many authors [19]. They have shown that the angular bicyclic structure is a determining factor for the reactivity. Moreover, by computer graphic techniques, it has been shown that the rings $\mathrm{A}$ and $\mathrm{C}$ of the molecules with comparable affinity for tubulin are at an angle of $50-60^{\circ}$ to each other [20]. In this way, we have already pointed out the influence of the steric hindrance for the binding of ethyl 5-amino-3phenylpyrido[3,4- $b]$ pyrazin-7-yl carbamate ligands to tubulin [5]. We have suggested that a weak increase in fluorescence intensity upon interaction with tubulin resulted from a more hindered rotation of the phenyl group, in accordance with the high values of the dihedral angle, and that, for $\mathrm{S}, \mathrm{T}$ and $\mathrm{U}$, the phenyl group was less anchored in the binding site.

\subsection{Dipole moments}

All the compounds had almost identical calculated dipole moments ranging from 4.01 to 4.31 Debye. As the dipole moment reflects the charges distribution, it is used as a theoretical descriptor in quantitative structure activity relationships (QSAR) to explain ligands binding. Verdier-Pinard et al. have found that, for a series of molecules inhibiting tubulin polymerization, the $x$-axis contribution to dipole moment is a good descriptor of the biological activity [21]. The small variation of the dipole moment does not explain the difference in the binding of the four compounds to tubulin.

\subsection{Energy levels}

The energies of the HOMO and of the LUMO orbitals are reported in Table 1. The ionization potential values are moderate and similar for all compounds. Moreover, the compounds are good electron donors. The HOMO are stabilized by the double bond at $\mathrm{N} 1=\mathrm{C} 2$ by a value of $0.3-0.4 \mathrm{eV}$ and poorly affected by the methyl group in $\mathrm{C} 2$. We observed that $\mathrm{S}$ and $\mathrm{V}$ compounds which have the most negative free energy values for binding to tubulin possess the less negative values of $E_{\text {Номо }}$ and $E_{\mathrm{LUMO}}$. From CNDO/2 results, such a qualitative relationship between the electron donor character of the ligands and the affinity to tubulin has been ruled out and the occurrence of a charge transfer with an amino acid on the tubulin chain has been previously discussed [5]. However, the experimental fluorescence spectra does not confirm a charge transfer with a tryptophanyl residue, classically studied, but the authors did not exclude a charge transfer with another residue. Some semi-empirical calculations are in progress.

In the case of molecular modeling studies of colchicine analogs Verdier-Pinard et al. have found that for tubulin polymerization inhibition the model with the greatest significance takes into account the energy of the LUMO [21]. This descriptor showed no obvious linear trend when plotted against activity but was required to "fine-tune" the model. So, it appears that HOMO and/or LUMO of the compounds are implicated in the mechanism of binding to tubulin. This will be understood when the primary structure of the tubulin binding site will be elucidated.

\subsection{HOMO and LUMO localizations}

Localizations of the atomic orbitals implicated in HOMO and LUMO were calculated from the coefficients of the $\mathrm{p}$ atomic orbitals. These densities are different of those calculated by the CNDO/2 method [5]. The AM1 method pointed out that the HOMO were to a great extend localized on the $\mathrm{C} 10-\mathrm{N} 4=\mathrm{C} 3$ moiety for the $\mathrm{S}$ and $\mathrm{V}$ compounds and on the ring $\mathrm{A}$ of the $\mathrm{T}$ and $\mathrm{U}$ compounds. Indeed, for the $\mathrm{S}$ compound the electrons of the $\mathrm{C} 10$ atom accounted for $30 \%$ in the HOMO (C3 for 14\%) while for T, the HOMO was for $27 \%$ localized on the $\mathrm{C} 8$ atom (13\% for $\mathrm{C} 10$ and $12 \%$ for the nitrogen atom at C5). The LUMO, for the four compounds were essentially localized on the rings $\mathrm{A}$ and $\mathrm{B}$. For $\mathrm{S}$ and $\mathrm{V}$, the electrons of C3, C7 and C9 accounted for 16, 18 and 10\%, respectively. While for $\mathrm{T}$ and $\mathrm{U}$, the electrons of C3, C7, C8 and N4 accounted for 19, 16, 10 and $12 \%$, respectively. The LUMO and HOMO are of the $\pi$-type. This is in agreement with the intensity of the lowest absorption energy bands with extinction coefficient superior to $5000 \mathrm{M}^{-1} \mathrm{~cm}^{-1}$ in hydrogen bond free solvents which suggests that the transition is due to a $\pi-\pi^{*}$ excitation. Clearly, the atomic orbitals of the phenyl ring slightly influenced the character of the HOMO and the LUMO. By contrast, MNDO results for colchicine have shown that $70-90 \%$ of the total electron density in the HOMO or LUMO is due to the tropolone ring [22]. Thus, for colchicine-binding to tubulin, it has been proposed that the colchicine ring $\mathrm{C}$ should bind first, followed by a slow 
Table 2

Mulliken charges of the studied compounds

\begin{tabular}{|c|c|c|c|c|}
\hline Atom & V & $S$ & $\mathrm{U}$ & $\mathrm{T}$ \\
\hline N1 & -0.330 & -0.289 & -0.111 & -0.182 \\
\hline $\mathrm{C} 2$ & -0.090 & 0.020 & -0.063 & -0.004 \\
\hline $\mathrm{C} 3$ & -0.033 & -0.027 & -0.036 & -0.032 \\
\hline N4 & -0.085 & -0.089 & -0.084 & -0.084 \\
\hline $\mathrm{C} 5$ & 0.240 & 0.242 & 0.226 & 0.220 \\
\hline N6 & -0.309 & -0.303 & -0.282 & -0.281 \\
\hline $\mathrm{C} 7$ & 0.228 & 0.225 & 0.186 & 0.184 \\
\hline $\mathrm{C} 8$ & -0.336 & -0.325 & -0.231 & -0.231 \\
\hline C9 & 0.188 & 0.157 & 0.044 & 0.051 \\
\hline $\mathrm{C} 10$ & -0.241 & -0.238 & -0.157 & -0.157 \\
\hline NH2 & -0.332 & -0.341 & -0.345 & -0.338 \\
\hline $\mathrm{NH}-\mathrm{R}$ & -0.295 & -0.296 & -0.288 & -0.289 \\
\hline
\end{tabular}

conformational change enabling the binding of ring A [23]. From the AM1 semi-empirical results on our molecules, if a $\pi$-stacking association between an aromatic amino acid of tubulin and ligands exists, as it is the case for colchicine analogs [22], it should appear in a second binding step. In the first step, according to the electron densities in the HOMO and LUMO, the rings $\mathrm{A}$ and $\mathrm{B}$ are involved. Kinetic studies have shown such a pathway for MDC, a bicyclic colchicine analog [24].

\subsection{Mulliken charge distribution}

The Mulliken charges are shown in Table 2. In the case of a tubulin charge driven binding, the differ-

Table 3

Wiberg bond orders of the studied compounds

\begin{tabular}{lllll}
\hline Bond & $\mathrm{V}$ & $\mathrm{S}$ & $\mathrm{U}$ & $\mathrm{T}$ \\
\hline $\mathrm{N} 1-\mathrm{C} 2$ & 0.95 & 0.95 & 1.66 & 1.61 \\
$\mathrm{~N} 1-\mathrm{C} 9$ & 1.15 & 1.12 & 1.19 & 1.22 \\
$\mathrm{C} 2-\mathrm{C} 3$ & 0.95 & 0.94 & 1.19 & 1.12 \\
$\mathrm{C} 3-\mathrm{N} 4$ & 1.80 & 1.82 & 1.58 & 1.58 \\
C3-C(a) (C6H5) & 0.99 & 0.98 & 0.99 & 0.98 \\
N4-C10 & 1.09 & 1.08 & 1.25 & 1.26 \\
C5-N6 & 1.34 & 1.34 & 1.45 & 1.45 \\
C5-C10 & 1.20 & 1.20 & 1.08 & 1.08 \\
C5-NH2 & 1.17 & 1.17 & 1.18 & 1.17 \\
N6-C7 & 1.33 & 1.33 & 1.24 & 1.23 \\
C7-C8 & 1.35 & 1.34 & 1.44 & 1.46 \\
C7-NH-R & 1.03 & 1.03 & 1.03 & 1.03 \\
C8-C9 & 1.30 & 1.32 & 1.25 & 1.23 \\
C9-C10 & 1.25 & 1.27 & 1.27 & 1.27 \\
\hline
\end{tabular}

ences observed in the distribution of the electron densities could be responsible for the differences observed for the $\Delta H_{0}$ (see Table 1). For the $\mathrm{S}$ and $\mathrm{V}$ compounds, according to AM1 calculations, the highest electron densities are on atoms N1, N6 and C8. For the $\mathrm{T}$ and $\mathrm{U}$ compounds, the highest negative net atomic charge is found on N6. Nitrogen atoms could primarily be considered as site of specific interactions with solvent and/or with other molecules (ligandreceptor interaction).

The differences observed in the charge distribution suggest that the properties of $\mathrm{S}$ and $\mathrm{V}$ on one hand and $\mathrm{T}$ and $\mathrm{U}$ on the other hand are different. For example, the compounds are supposed to exhibit different protonation sites. This fact will be further investigated in a manuscript in preparation. However, preliminary AM1 results indicated that for $\mathrm{S}$ the protonation occurs on the N6 atom, while in the case of T, protonation on $\mathrm{N} 4$ atom is energetically favored.

The effect of the double bond between the atoms $\mathrm{N} 1$ and $\mathrm{C} 2$ on the electron density distribution is calculated by the difference of the atomic charges between $\mathrm{V}$ and $\mathrm{U}$. As seen previously, the effect is important for $\mathrm{N} 1$, and is spread through the other atoms in particular in C8 $(+0.105)$, C9 $(-0.144)$ and $\mathrm{C} 10(+0.084)$. Concerning the introduction of the $\mathrm{CH}_{3}$ substituent at atom $\mathrm{C} 2$, the effect on the charge distribution could be calculated from the results of $\mathrm{S}$ and $\mathrm{V}$, for example. As expected, we noted an important effect on atom $\mathrm{C} 2(+0.110)$ and a moderate one on $\mathrm{N} 1(+0.041)$, but the electron density is almost the same on C3 atom $(+0.006)$. Instead, the $\mathrm{C} 9$ atom becomes more negative $(-0.031)$.

\subsection{Wiberg bond orders}

The Wiberg bond orders are reported in Table 3. For all molecules, the ring A bonds were conjugated. Indeed the mean calculated bond value is about 1.34. It is not the case for ring $\mathrm{B}$, the results clearly indicate that the bonds between N4-C10 and C3-C(a) of the compounds V and $\mathrm{S}$ are of $\sigma$-type. This result was expected and is due to the high values of the dihedral angle between rings $B$ and $\mathrm{C}$ inhibiting the delocalization of the electrons through the $\mathrm{C} 3-\mathrm{C}$ (a) bond. Moreover, for the four molecules the Wiberg bond order value of the $\mathrm{C} 3-\mathrm{C}(\mathrm{a})$ bond remains constant at 0.99 which indicates a pure $\sigma$-bond 
Table 4

UV-visible spectra parameters in neutral aqueous buffer, $\lambda_{\max }$ and $\epsilon$, and the semi-empirical results: $\lambda_{\text {calc }}$, oscillator strength length, and state dipole (Debye) (calculated transitions with oscillator strength length values inferior to $4 \times 10^{-2}$ are omitted)

\begin{tabular}{|c|c|c|c|c|c|}
\hline & $\lambda_{\max }(\mathrm{nm})$ & $\epsilon\left(\mathrm{M}^{-1} \mathrm{~cm}^{-1}\right)$ & $\lambda_{\text {calc. }}(\mathrm{nm})$ & Osc. str. length & State dipole (Debye) \\
\hline \multirow[t]{6}{*}{ V } & 238 & 29887 & 232 & 0.1389 & 5.3069 \\
\hline & & & 237 & 0.2880 & 10.5622 \\
\hline & 265 & 17540 & 259 & 0.3997 & 7.6165 \\
\hline & 355 & 9425 & 262 & 0.1409 & 5.8916 \\
\hline & & & 321 & 0.0423 & 9.2081 \\
\hline & 390 & 5432 & 368 & 0.6619 & 3.696 \\
\hline \multirow[t]{4}{*}{ S } & 240 & 29888 & 237 & 0.1648 & 4.1172 \\
\hline & 265 & 14787 & 252 & 0.4787 & 6.0613 \\
\hline & 347 & 7939 & 316 & 0.0840 & 9.0397 \\
\hline & 384 & 10404 & 351 & 0.5932 & 3.7128 \\
\hline \multirow[t]{5}{*}{$\mathrm{U}$} & 250 & 13480 & 252 & 0.0417 & 7.3154 \\
\hline & 294 & 25486 & 270 & 0.8849 & 5.1608 \\
\hline & & & 277 & 0.0504 & 6.3263 \\
\hline & 332 & 9912 & 319 & 0.3768 & 3.9986 \\
\hline & 409 & 6665 & 378 & 0.3189 & 2.1594 \\
\hline \multirow[t]{4}{*}{$\mathrm{T}$} & 263 & 17618 & 272 & 0.9099 & 4.9935 \\
\hline & 284 & 15954 & 277 & 0.0408 & 6.3986 \\
\hline & 321 & 8247 & 317 & 0.3085 & 4.1506 \\
\hline & 391 & 5479 & 372 & 0.3340 & 2.1761 \\
\hline
\end{tabular}

without conjugation. For compounds $\mathrm{T}$ and $\mathrm{U}$ with a double bound between $\mathrm{N} 1$ and $\mathrm{C} 2$, the Wiberg bond order is about 1.6 indicating that this bond is of $\pi$-type and weakly conjugated. Finally, the $\mathrm{C} 7-\mathrm{NH}$ Wiberg bond order value is about 1.0 for all the four compounds. No electron delocalization exists between ring $\mathrm{A}$ and the side chain, the rotation around the $\mathrm{C} 7-\mathrm{NH}$ bond is completely free.
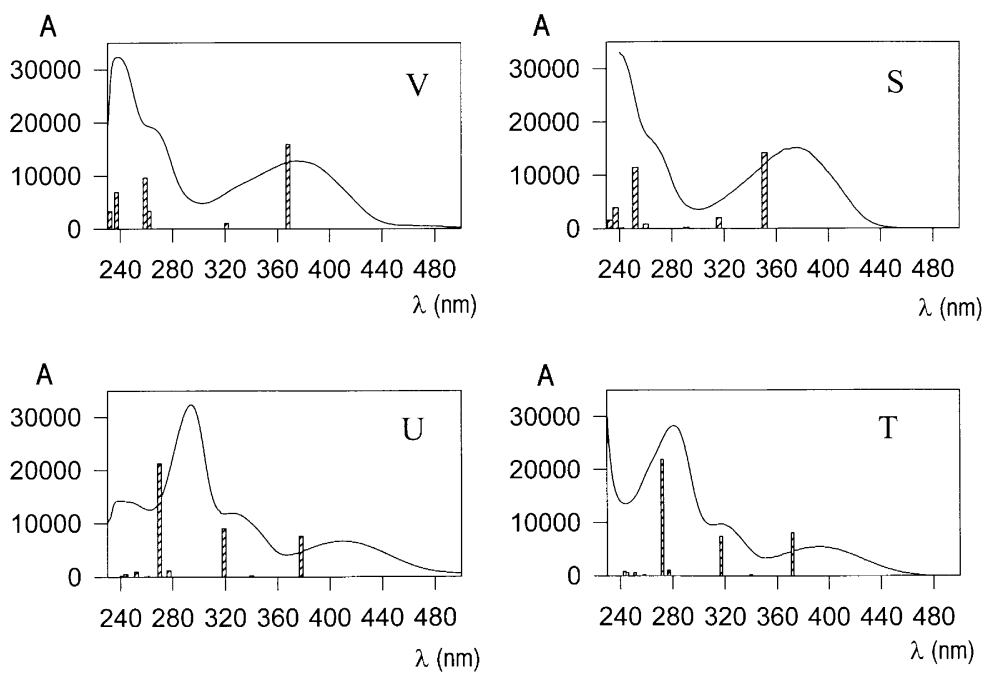

Fig. 2. Experimental absorption spectra of the ligands and calculated transitions (the amplitudes of the calculated transitions are drawn proportional to the oscillator strength lengths. 
Table 5

Absorption band maxima and fluorescence band maxima of the studied compounds in sodium phosphate buffer $\mathrm{pH} 7$, free and bound to tubulin, and calculated Stockes shifts

\begin{tabular}{llll}
\hline & Absorption $\lambda_{\max }(\mathrm{nm})$ & Fluorescence $\lambda_{\max }(\mathrm{nm})$ & Stokes shift $\left(\times 10^{3} \mathrm{~cm}^{-1}\right)$ \\
\hline Free & & & \\
V & 390 & 488 & 10.2 \\
S & 384 & 477 & 10.8 \\
U & 409 & 515 & 9.4 \\
T & 391 & 520 & 7.8 \\
Bound & & & \\
V & 410 & 490 & 12.5 \\
S & 400 & 465 & 15.4 \\
U & 410 & 503 & 10.8 \\
T & 392 & 492 & 10.0 \\
\hline
\end{tabular}

\subsection{Absorption and emission}

The measured and deconvoluted UV-visible spectra parameters in neutral aqueous buffer, $\lambda_{\max }$ and $\epsilon$, and the semi-empirical calculations: $\lambda_{\text {calc }}$, oscillator strength length, state dipole, transitions and CI coefficients are reported in Table 4.

Measured absorption UV-visible spectra and calculated transitions are shown in Fig. 2. Although the calculated frequencies are generally blue shifted, the measured absorption spectra are reproducible. The introduction of a double bond in the $\mathrm{N} 1-\mathrm{C} 2$ position induces a red shift in the position of the calculated low-energy band. This shift is about $21 \mathrm{~nm}$ for $\mathrm{T}$ with respect to $S$ and $10 \mathrm{~nm}$ for $U$ with respect to $V$. Experimentally, the red shift is higher for $U(19 \mathrm{~nm})$ than for $\mathrm{T}(7 \mathrm{~nm})$. Higher conjugation and resonance effects between $\pi$-clouds of rings are generally evoked to explain a red shift. Concerning the phenyl ring, a stronger conjugation is obtained by decreasing the dihedral angle values. Indeed, for the $\mathrm{S}$ compound calculations with a planar conformation in comparison with a twisted one, indicated a red shift of $30 \mathrm{~nm}$ for the low-energy absorption band (not shown). However, for S and T compounds we obtained optimized conformations with the same dihedral angle value, and we observed an increase in the dihedral angle value from $\mathrm{U}$ to $\mathrm{V}$. AM1 calculations indicated that substitution by a methyl group at $\mathrm{C} 2$ induces a blue shift of $17 \mathrm{~nm}$ for $\mathrm{S}$ compared to $\mathrm{V}$ and $6 \mathrm{~nm}$ for $\mathrm{T}$ compared to U. Surprisingly, the measured and deconvoluted spectra presented smaller shifts for $\mathrm{S}$ $(6 \mathrm{~nm})$ than for $\mathrm{T}(18 \mathrm{~nm})$. So, the discrepancy between the calculated and the measured $\lambda_{\max }$ on one hand and their variations on the other hand could be due to some interactions with the solvent, for example hydrogen bonds, affecting the theoretical conformation. Peyrot et al. [8] have pointed out that in water, specific solvent effect takes place on compounds with a 1,2-dihydro structure ( $\mathrm{S}$ and V) but not on compounds with a double bond between $\mathrm{N} 1$ and $\mathrm{C} 2$ and they concluded that an hydrogen bond was created between N1 and water. For all the four compounds, in the lowest energy excited state, the calculated dipole moment values are smaller than the values of the ground state (compare Tables 1 and 4). In solvents of decreased polarity, it is well known that the absorption band is shifted to longer wavelength (low energy) [25]. Indeed, S and V displayed a shift to longer wavelength (Barbier results not published). For $\mathrm{T}$ and $\mathrm{U}$ this is not observed, the lower energy band in solvents of various polarities did not shift. The UV-visible absorption spectra of the S and $\mathrm{V}$ compounds developed red shifts and vibrational structures upon binding to tubulin [8]. For $\mathrm{T}$, no significant variation is observed when it binds to tubulin. For U, only a $5 \mathrm{~nm}$ red shift is observed in the $332 \mathrm{~nm}$ band. Then, according to the small values of the excited state dipole moments and to the red shifts observed in the solvents with small polarity indexes, the observed modifications on absorption spectra upon binding to tubulin could be interpreted as due to reduced polarity in tubulin binding site.

Each of the four ligands in neutral aqueous buffer has a slight fluorescence emission. Whatever the solvent, the fluorescence spectra have a thinner 
<smiles>[R7]C1([R2])Nc2cc(NC(=O)OCC)[nH]c(=N)c2N=C1c1ccccc1</smiles><smiles>[R2]C1([R2])Nc2c/c(=N/C(=O)OCC)[nH]c(N)c2N=C1c1ccccc1</smiles>

imino form II

Fig. 3. Structures of the imino I and II forms of S.

shape than the absorption spectra. For example, the fluorescence emission spectrum of $\mathrm{V}$ in neutral aqueous buffer may be deconvoluted as a sum of three bands positioned at 434, 459 and $489 \mathrm{~nm}$. The FWHM are 21, 34 and $47 \mathrm{~nm}$, respectively. The deconvoluted absorption spectrum produced three bands centered at 265, 354 and $389 \mathrm{~nm}$ with calculated FWHM of 33, 97 and $54 \mathrm{~nm}$. The FWHM of the fluorescence spectra decreased in polar/protic solvents. This suggests that the four ligands are more planar in their first excited singlet state $\left(S_{1}\right)$ than in the ground state $\left(\mathrm{S}_{0}\right)$ and their planarity increases in the polar/protic solvents. In all the cases, the Stokes' shift values calculated for the free ligands in neutral aqueous buffer (see Table 5) are

Table 6

Total energies of the imino forms minus total energies of the amino forms, $\Delta E$, dipole moments, $\mu$, energies of the HOMO, $E_{\text {Номо, and }}$ energies of the LUMO, $E_{\mathrm{LUMO}}$ for the imino I and II forms calculated using AM1

\begin{tabular}{lrlll}
\hline & \multicolumn{1}{c}{$\Delta E$} & $\mu$ (Debye) & $E_{\text {НОМО }}(\mathrm{eV})$ & $E_{\text {LUMO }}(\mathrm{eV})$ \\
\hline V I & 18.23 & 6.94 & -7.6229 & -0.4991 \\
V II & 6.88 & 5.32 & -8.0638 & -0.5695 \\
S I & 19.30 & 6.82 & -7.6202 & -0.4778 \\
S II & 8.18 & 5.50 & -8.0461 & -0.5538 \\
U I & 15.58 & 4.09 & -8.3346 & -0.9102 \\
U II & 15.79 & 6.45 & -8.0653 & -1.1713 \\
T I & 15.14 & 4.10 & -8.3389 & -0.7943 \\
T II & 14.63 & 6.38 & -8.0515 & -1.1245 \\
\hline
\end{tabular}

higher, particularly for $\mathrm{S}$ and $\mathrm{V}$. The effect is even more important when the compounds are bound to tubulin. This indicates that the geometries of the ligands in the $S_{0}$ and $S_{1}$ states are different from each other and that the difference increases upon binding to tubulin. Furthermore, large values of the observed Stokes' shifts indicated that an excited state rearrangement took place. We could think that the rotation of the phenyl ring $\mathrm{C}$ resulting in the formation of a flattened structure is responsible for the fluorescence with large Stokes' shift. However, the variation of energy for a totally planar structure calculated by AM1 is about $75 \mathrm{kcal} / \mathrm{mol}$ for the $\mathrm{S}$ compound (not shown). So, the rotation of the phenyl ring could be only moderate and appears to be an unfavorable pathway.

\subsection{The imino forms}

Several canonical forms may be proposed for the four compounds. Among them, two imino forms I and II are shown in Fig. 3. These resonance structures are stabilized by an intramolecular hydrogen bond between the COO moiety of the side chain and a hydrogen of the N6 atom. The side chain adopts a more planar configuration and its mobility is reduced. However, these forms are predicted by AM1 to be thermodynamically less stable than the amino forms of Fig. 1 (see Table 6 for the total energies and dipole moments). Nevertheless, their dipole moments are higher than the dipole moments of the amino forms. Then, their stability would further increase with the polarity of the solvents. For the compounds with 1,2dihydro structure ( $\mathrm{S}$ and $\mathrm{V}$ ), the imino II form is thermodynamically more stable than the imino I form, the difference of energy between the amino and the imino II forms is only $8.2 \mathrm{kcal} / \mathrm{mol}$ for $S$ and $6.8 \mathrm{kcal} / \mathrm{mol}$ for $\mathrm{V}$. This indicated that, for these compounds, either both amino and imino II forms, represent the population of the fundamental state $\mathrm{S}_{0}$, or, that the imino II forms represent the $S_{1}$ state. Indeed, UV-visible absorption spectra and the $\lambda_{\text {calc }}$ values for $\mathrm{V}$ and $\mathrm{S}$ in the imino form II agree rather well with the experimental $\lambda_{\max }$ values (see Fig. 4 and compare with Fig. 2 ). For the compounds with a double bond between $\mathrm{N} 1$ and C2, imino I and II forms exhibit similar instability, their calculated energies are higher than the energies of the amino forms by about $15 \mathrm{kcal} / \mathrm{mol}$. 
A

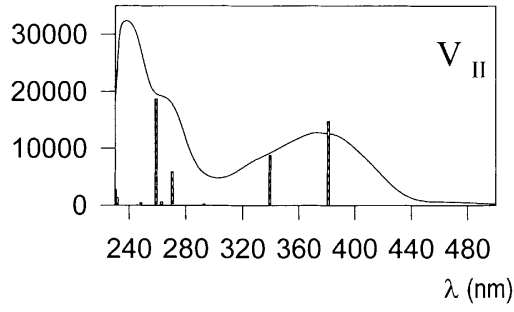

A

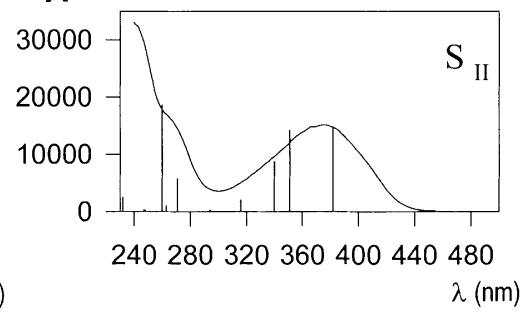

Fig. 4. Experimental UV-visible spectra of $\mathrm{V}$ and $\mathrm{S}$ and calculated transitions for the imino II forms.

Therefore, it is unlikely that these forms exist in the fundamental state $\mathrm{S}_{0}$. So, the compounds $\mathrm{T}$ and $\mathrm{U}$ preferentially adopt the amino form in the ground state. Moreover, no agreement is found between the experimental and the calculated absorption spectra for the ligands in the imino I or II form. This means that these forms are not involved in the $S_{1}$ state. However, a possibility exists that the imino forms are representative of some transition state leading to the $S_{1}$ state.

The dipole moment (Table 6) reflects the heterogeneous charge distribution within the molecule and to some extent the electrostatic potential around the molecule. The later reveals that the molecular electro-
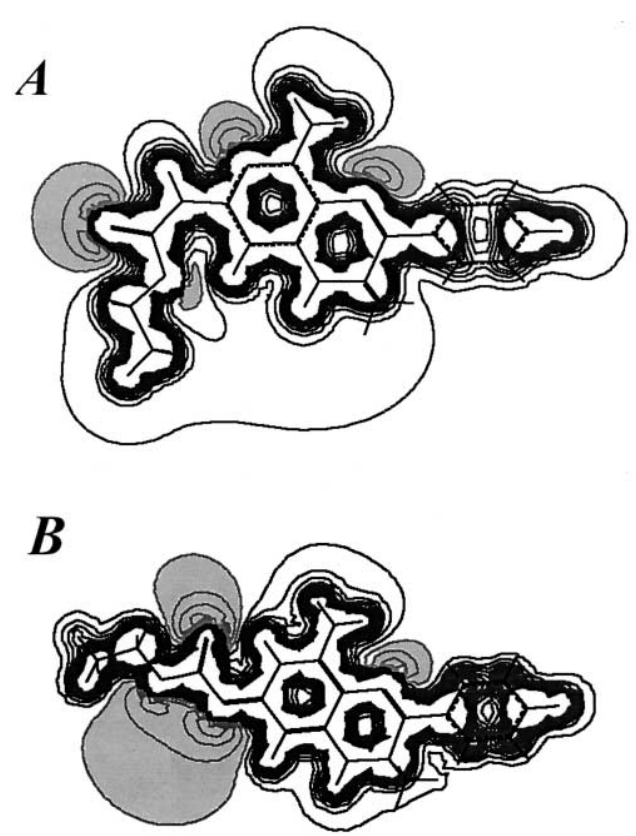

Fig. 5. The distribution of MEP (negative potential regions are greyed) for the $\mathrm{S}$ compound in the plane of rings $\mathrm{A}$ and $\mathrm{B}$. $A$, calculated for the amino form. $B$, calculated for the imino II form. static potential (MEP) is deeply modified (see Fig. 5 for amino and imino II forms of S). Whereas, the amino MEP map of S shows three negative potential regions located on one side of the molecule, the imino II MEP map of $\mathrm{S}$ shows an unmodified negative region near atom N4 and two wide negative potential regions on both sides of the heteratoms of the side chain at C7. This feature may be of tremendous importance for binding to tubulin since MEP maps are key signature which, for example, permits the distinction between active and inactive anticonvulsant compounds [26].

The ionization potential and electron affinity of imino II forms of S and V compounds remain in the same order of calculated values for amino forms of $\mathrm{S}$ and $\mathrm{V}$ molecules. However, the electron densities in the HOMO and LUMO of the imino I and II forms are different, as expected, from the calculated densities of the amino forms. For the imino II $\mathrm{S}$ compound, the electron density in $\mathrm{C} 10$ account for $25 \%$ in the HOMO (C8 for $21 \%, \mathrm{C} 3$ for $10 \%$ and the nitrogen atom of the side chain at $\mathrm{C} 7$ for $15 \%$ ). In comparison with the calculated values for the amino $\mathrm{S}$, the participation of $\mathrm{C} 10$ decrease and $\mathrm{C} 8$ has a higher density than in the case of ligand T. Now, in the LUMO orbital of imino II form of $\mathrm{S}$, the electron densities of $\mathrm{C} 3, \mathrm{C} 5$ and N4 account, respectively, for 15, 15 and $12 \%$. A large participation of the atoms $\mathrm{C} 3$ and $\mathrm{N} 4$ is observed for the amino form of the T compound. We conclude that the HOMO and LUMO orbitals of the imino II forms of $\mathrm{S}$ and $\mathrm{V}$ mimic the amino forms of the $\mathrm{T}$ and U compounds.

\section{Conclusions}

In this work, AM1 calculations of ground-state 
parameters of the ethyl 5-amino-3-phenylpyrido[3,4$b$ ]pyrazin-7-yl carbamate ligands allowed a better understanding of the behavior of each drug and enlightened their mechanism of binding to tubulin. Thus, Mulliken charge distribution indicates that the electron density is distributed on atoms N1, N6 and C8 for S and $\mathrm{V}$ compounds. For $\mathrm{T}$ and $\mathrm{U}$ the electron density is preferentially distributed on atom N6. For the four compounds the AM1 calculated dipole moment are highly similar and cannot explain the difference reported upon binding to tubulin. The largest dihedral angle calculated values between the rings $\mathrm{B}$ and $\mathrm{C}$ for $\mathrm{S}$ and $\mathrm{T}$ are explained by the presence of a methyl group in $\mathrm{C} 2$. Wiberg bond order demonstrated that the bond between N4-C10 is of $\sigma$-type for $\mathrm{S}$ and $\mathrm{V}$ compounds. For the dehydrocompounds ring B is weakly conjugated. For all the compounds the bond between $\mathrm{C} 3-\mathrm{C}(\mathrm{a})$ is of $\sigma$-type: there is no electron delocalization between ring $\mathrm{B}$ and phenyl C. Moreover, the energy levels, HOMO and LUMO localizations indicated that the atomic orbitals of rings $\mathrm{A}$ and $\mathrm{B}$ are particularly implicated in the mechanism of binding to tubulin, and suggested that rings $\mathrm{A}$ and $\mathrm{B}$ mainly participate in the first step of binding to tubulin, the second step consisting in the interaction of the phenyl ring $\mathrm{C}$.

The theoretical UV-visible absorption spectra are somewhat different from the experimental spectra, specially at low energy. This discrepancy may be due to specific solvent effect, such as hydrogen bonding. Analysis of the fluorescence emission spectra and comparison with the absorption spectra indicated that the geometries of the ligands in the $S_{0}$ and $S_{1}$ states are very different from each other, and this difference is more important upon binding to tubulin.

Furthermore, for the four compounds several resonance structures are proposed. For S and V compounds, both amino and imino II canonical forms could represent the population of the fundamental state $S_{0}$ or the imino II form could represent the $S_{1}$ state. For $T$ and $U$ compounds, the theoretical calculations indicated that the imino form is highly improbable. Interestingly, the HOMO and LUMO orbitals of the imino II form of S and $\mathrm{V}$ mimics those of $\mathrm{T}$ and $\mathrm{U}$ compounds.

\section{Acknowledgements}

We are grateful to Dr Daniel Laffite, Dr Claude
Aubert and Mrs Mireille Coqueron for useful and helpful discussions.

\section{References}

[1] S.B. Hastie, Pharmacol. Ther. 51 (1991) 377.

[2] D. Leynadier, V. Peyrot, M. Sarrazin, C. Briand, J.M. Andreu, G.A. Rener, C. Temple Jr., Biochemistry 32 (1993) 10675.

[3] C. De Ines, D. Leynadier, I. Barasoain, V. Peyrot, P. Garcia, C. Briand, G.A. Rener, C. Temple Jr., Cancer Res. 54 (1994) 75.

[4] Y. Engelborghs, A. Van de Candelaere, Cell. Pharmacol. (suppl. 1) (1993) S23-S26.

[5] P. Barbier, V. Peyrot, C. Dumortier, A. D'Hoore, G.A. Rener, Y. Engelborghs, Biochemistry 35 (1996) 2008.

[6] P. Barbier, V. Peyrot, M. Sarrazin, G.A. Rener, C. Briand, Biochemistry 34 (1995) 16821.

[7] N. Allam, J.M. Millot, D. Leynadier, V. Peyrot, C. Briand, C. Temple Jr., M. Manfait, Int. J. Macromol. 17 (1995) 55.

[8] V. Peyrot, P. Barbier, M. Sarrazin, C. Briand, J.M. Andreu, Photochem. Photobiol. 70 (1999) 710.

[9] Hyperchem, Hypercube, Inc., Publication HC50-01-01-00, September 1996, Canada.

[10] N.A. Allinger, J. Am. Chem. Soc. 99 (1977) 8127.

[11] M.A. Thompson, M.C. Zerner, J. Am. Chem. Soc. 113 (1991) 8210.

[12] M.A. Thompson, E.D. Glendening, D. Feller, J. Phys. Chem. 98 (1994) 10465.

[13] M.A. Thompson, G.K. Schenter, J. Phys. Chem. 99 (1995) 6374.

[14] M.A. Thompson, J. Phys. Chem. 100 (1996) 14492.

[15] J. Ridley, M. Zerner, TCA (Berl.) 32 (1973) 111.

[16] M.C. Zerner, G.H. Loew, R.F. Kirchner, U.T. MuellerWesterhoff, J. Am. Chem. Soc. 102 (1980) 589.

[17] M.C. Zerner, in: K.B. Libkowitz, D.B. Boyd (Eds.), Semiempirical Molecular Orbital Methods, Reviews in Computational Chemistry, vol. II, VCS, 1991 (chap. 8).

[18] Sigmaplot, SPSS Inc, 1997, USA.

[19] H.G. Capraro, A. Brossi, in: A. Brossi (Ed.), The AlkaloÏds, vol. 23, Academic Press, New York, 1984 (chap. 1).

[20] A.T. Mc Gown, B.W. Fox, Anticancer Drug Des. 3 (1989) 249.

[21] P. Verdier-Pinard, J.-Y. Lai, H.-D. Yoo, J. Yu, B. Marquez, D.G. Nagle, M. Nambu, J.D. White, J.R. Falck, W.H. Gerwick, B.W. Day, E. Hamel, Mol. Pharmacol. 53 (1998) 62.

[22] S.B. Hastie, R.P. Rava, J. Am. Chem. Soc. 111 (1989) 6993.

[23] J.M. Andreu, S.M. Timasheff, Biochemistry 21 (1982) 532.

[24] C. Dumortier, J.L. Potenziano, S. Bane, Y. Engelborghs, Eur. J. Biochem. 249 (1997) 265.

[25] J.R. Lakowicz, Principles of Fluorescence Spectroscopy, Plenum Press, New York, 1983.

[26] J. Obniska, A. Zejc, J. Karolak-Wojciechowska, Il Farmaco 54 (1999) 423. 